\title{
A Função de Controle nos Sistemas Integrados de Manufatura
}

\begin{abstract}
RESUMO:Faz-se uma proposição de vários conceitos atinentes ao gerenciamento da produção, muitos dos quais são válidos também para os Sistemas de Manufatura Convencional, mas que estão aqui colocados dentro do contexto dos Sistemas Integrados de Manufatura (SIMs), que são os sistemas desenvolvidos sob a filosofia de fabricação CIM ('Computer Integrated Manufacturing = Manufatura Integrada por Computador). Mostra-se que a integração depende basicamente da função controle.
\end{abstract}

ABSTRACT: Manufacturing integration is reviewed and various concepts and definitions founded in literature are presented for conventional and automated manufacturing systems.

The survey emphasizes information system management and control importance in order to improve manufacturing functions integration.

palavras-chave: sistemas de produção, manufatura integradas, gerência da produção

Key words: production systems, integrated manufacturing, production management

\section{Flavio Cesar F. Fernandes}

Professor Assistente DEP-UFSCar. Mestre em Engenharia de Produção pela EPUSP.

Doutorando em Engenharia de Fabricação na EESC-USP.

Departamento de Engenharia de Produção

Universidade Federal de São Carlos - UFSCar

Via Washington Luiz, km. 23513560 - São Carlos - SP 


\section{Introdução}

Com o desenvolvimento da tecnologia de informação, o computador tem se tornado um elemento catalisador da integração das funções desempenhadas no sistema fabril: integração do projeto do produ to com o planejamento do processo, este com o gerenciamento da produção, este com a fabricação, etc., e como agentes dessa catalisação aparecem os SistemasdeInformação (SI), que são subsistema do Sistema de Controle. (Há os que colocam a seguinte relação, Sistema de Controle $=\mathrm{SI}+$ processo decisório.)

Isso pode ser constatado na literatura recente. Por exemplo, em Hitomi [13], a Tecnologia de Fabricaçãoéincorporada à Tecnologia de Gerenciamento. Hitomi entende que a tecnologia de fabricação trata do fluxo de materiais, incluindo-se aí a transformação dos materiais, enquanto que a tecnologia de gerenciamentolida principalmentecom ofluxo de informações deforma a gerenciar efetivamente ofluxo de materiais através do planejamento e de controle. Os capítulos do livrode Hitomi se sucedem em ordem compatível com as próprias etapas naturais do desenvolvimento dos Sistemas de Fabricação (SF). Tem-se os princípios dos $\mathrm{SF}$ e o processo; o gerenciamento da produçãoe a otimização dasatividades de gerenciamento; a automaçãocom as tecnologias do tipo 'computer-aided' e o Sistema de Informação (SI) que é um requisito para a obtenção de um SF integrado (sem SI não há controle, sem controle não há integração).

Como no ambiente integrado todos os aspectos devem ser levados em conta, o volume de dados é alto e deve ser alta a qualidade da transformação desses dados em informações úteis. Portanto o computador é uma ferramenta essencial e oSistema de Informação o agente do processo de integração.

Os FMSs (Sistemas Flexíveis de Manufatura) no geral estão presentes nos SIMs.
Para Ferdows e Lindberg [8], mais do que um investimento em tecnologia, a implantação de FMSs revela uma maneira mais profícua de pensar e gerenciar a manufatura, colocando a manufatura num papel estratégico dentro da empresa. Não tem sentido, ou seja, não deve haver implantação de FMSs num ambiente em que a filosofia CIM (Manufatura Integrada por Computador) de fabricação não tenha sidoadotada. Pela pesquisa que realizaram, Ferdows e Lindberg [8] concluíram: “aqueles que estão enfatizando FMS também estão dando ênfase a muitas outras idéias avançadas nogerenciamento da produção".

Merchant [17] previu que sistemas computadorizados para a completa automação e otimização da fabricação de peças estariam totalmente desenvolvidos entre 1980 e 1985. Mesmo que a sua previsão ainda não esteja totalmente confirmada, não há dúvida de que os esforços e desenvolvimentos apontam nessa direção.

Tudo isso está ocorrendo nos Sistemas Integrados de Manufatura (SIMs), que são os sistemas desenvolvidos sob a filosofia de fabricação CIM ('Computer IntegratedManufacturing') - Manufatura Integrada por Computador.

A faixa de aplicação do CIM é intermediária, aproximadamente de médio volume e de média variedade (veja Figura 1).

Nos SIMs, a caríssima tecnologia da automação está presente e, para se obter resultados compensadores, há necessidade de se obter integração entre os cincos componentes do sistema, a saber: 'hardware', 'software', gerenciamento da base de dados, tecnologia da comunicação e recursos humanos. Para Singhal et alii [19], atualmente é virtualmente impossível projetar e instalar um sistema desses onde cada um dos cinco subsitemas possa completamente explorar os outros quatro. Isso naturalmente configura um campo motivador de pesquisas. 


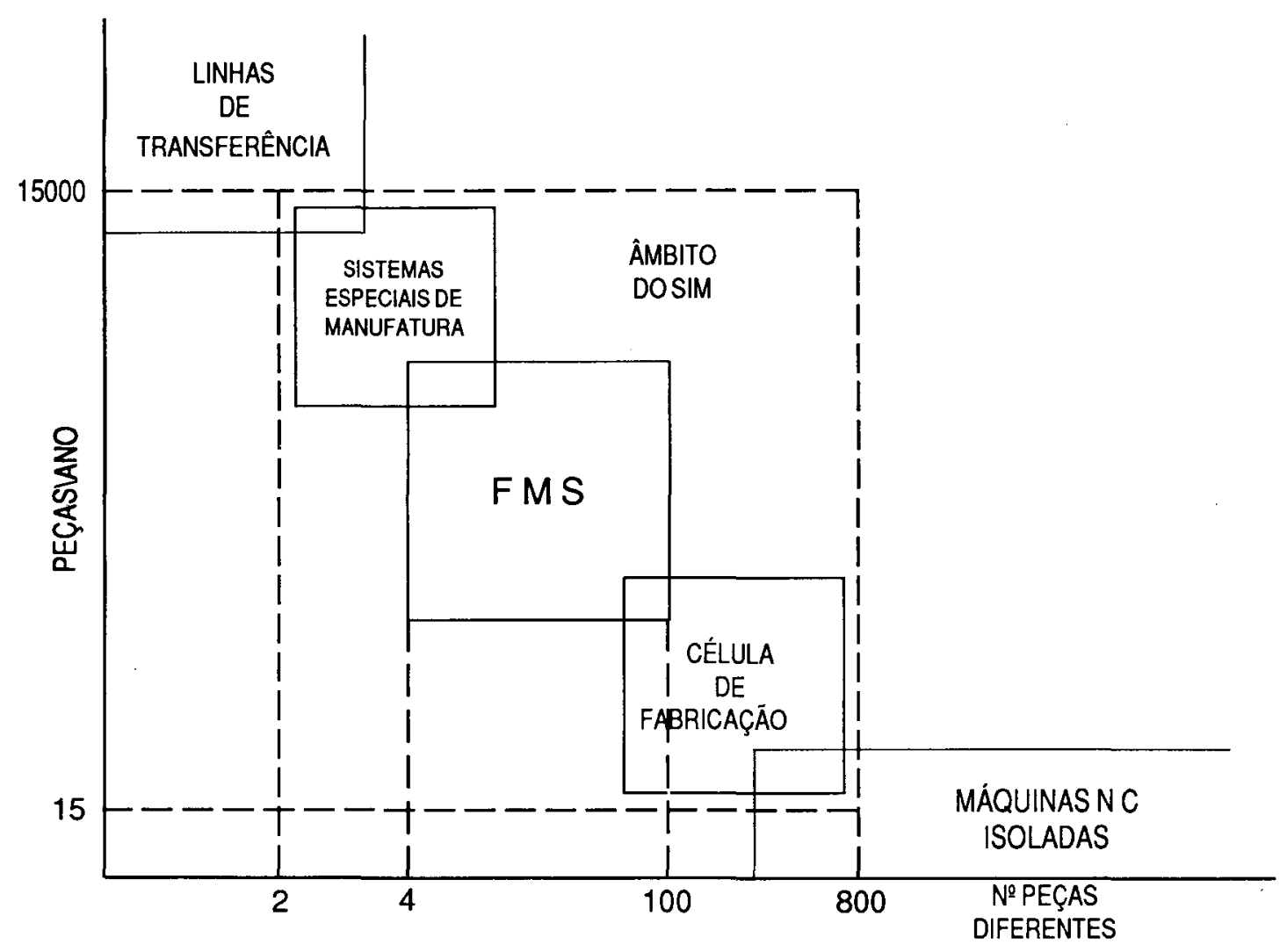

Figura 1. O âmbito do SIM adaptado de Groover [11].

Uma evidência de que a Manufatura Atual está se tornando complexa e por outro lado 'chic', é a explosão de siglas com as quais o leitcr deve se familiarizar. Outra observação é que não fazemos questão de traduzir termos em inglês que já se impregnaram na linguagem da manufatura: 'job-shop', 'flowshop', 'scheduling', 'computer-aided', etc.

\section{Visão Geral Sobre o SIM}

O SIM, Sistema Integrado de Manufatura, é a fábrica do futuro, e por sinal um futuro que já está se tornando presente nos países mais desenvolvidos. É a fábrica onde graças à automação flexível, com poucas pessoas se consegue um alto volume de produção pela fabricação de média variedade de produtos feitos em quantidades moderadas.

O SIM teve, como causa de seu aparecimento, mudanças no mercado produto (aumento da competição, introdução de novas tecnologias), no mercado consumidor (procura de produtos diversificados, diferenciados e renovados num curto intervalo de tempo) e na sociedade em geral (insatisfação com o trabalho nas fábricas, maior interesse por empregos no setor de serviços, etc.), e, como catalisador de seu desenvolvimento, tem-se as inovações tecnológicas (tecnologias do tipo 'computer-aided', redes locais de interligação (LAN), etc.) e as inovações metodológicas (o CIM, que é uma filosofia de fabricação baseada no enfoque sistêmico e na existência da tecnologia da informação.). 
Como tecnologias do tipo 'computeraided' compreende-se:

CAD ('computer-aided design') que "pode ser definido como o uso dos sistemas computacionais para auxiliar na criação, modificação, análise ou otimização de um projeto" [12];

CAM ('computer-aided manufacturing') que "pode ser definido como o uso dos sistemas computacionais para planejar, gerenciar e controlar as operações de uma instalação de manufatura através de interface direta ou indireta do computador com os recursos produtivos da instalação" [12];

DNC ('direct numerical control') envolve o uso de um computador de certo porte para controlar um certo número de máquinas NC ('numerical control') separadas;

CNC ('computer numerical control'), um computadoré usado para controlar uma máquina-ferramenta;

CAPP ('computer-aided process planning') é o planejamento do processo auxiliado por computador;

CPC ('computer process control') é o controle do processo por computador;

MRP II ('materials resource planning') "possui duas características que o distancia do MRP ('material requirement planning'):

\section{1. é um sistema financeiro e operacional}

2. é um simulador

Por ser simulador está apto a responder a questões do tipo: o que aconteceria se..." [12];
Armazenagem automatizada ('automated warehouse');

Movimentação de materiais automatizada, através de AGVs ('auto guided vehicles') que são veículos controlados por computador;

Robôs industriais que são manipuladores reprogramáveise controlados por computador;

$C A Q C$, controle de qualidade auxiliado por computador,

etc.

A eficácia do emprego dessas tecnologias pode ser aumentada utilizando-se algumasestratégias de controle da produção, tais como: JIT ('just-in-time'), GT (tecnologia de grupo) e OPT (tecnologia da produção otimizada).

Essas estratégias são baseadas emalguns princípios.

Entre os princípios do JIT se destacam a produção com zero defeito, zero atraso, zero estoque, zero quebra e zero papel (os 5 zeros).

Os princípios da GT são:

a) formação de famílias de peças semelhantes em termos de projeto e/ou processo;

b) formação de grupos de equipamentos cada um dos quais, na medida do possível, fabricando uma família de peças.

Alguns dos princípios da OPT são: o ótimo local (um subsistema qualquer) pode não contribuir para o ótimo global (sistema como um todo); balancear o fluxo e não a capacidade, etc. [1]. 
Já o enfoque sistêmico é a abordagem onde

* o modo de pensar

* o centro de atenção

* o método de resolver problemas

DEIXOU DE SER
primeiro analítico depois
sintético a parte
dividir em problemas
menores, resolver cada um,
juntar as soluções

PASSOU A SER

primeiro sintético depois analítico o todo

resolver o problema, decompor a solução
Essa abordagem estabelece que: o desempenho do todo depende do entrosamento entre as partes, e não apenas do desempenho de cada uma, ou seja, o melhor funcionamento do todo não corresponde, via de regra, somente ao melhor funcionamento de cada parte isoladamente.

As principais diferenças entre a Filosofia de Fabricação Convencional e a Filosofia de Fabricação CIM estão colocadas na Figura 2, tendo como fonte a referência [5].

\begin{tabular}{|c|c|}
\hline \multicolumn{2}{|c|}{ FILOSOFIA DE MANUFATURA } \\
\hline CONVENCIONAL & CIM \\
\hline \multicolumn{2}{|c|}{ DIVISÃO DO TRABALHO } \\
\hline $\begin{array}{l}\text { O Maior possível, o que acarreta: } \\
\text { - trabalho simples com a menor categoria } \\
\text { de salário possível; } \\
\text { - baixo envolvimento do trabalho; } \\
\text { - muitos pontos de interfaceamento. }\end{array}$ & $\begin{array}{l}\text { O Menor possivel, o que acarreta: } \\
\text { - trabalho qualificado com pessoal o mais } \\
\text { qualificado possível; } \\
\text { - alto envolvimento do trabalho; } \\
\text { - poucos pontos de interfaceamento. }\end{array}$ \\
\hline \multicolumn{2}{|c|}{ EXECUÇÃO DO TRABALHO } \\
\hline $\begin{array}{l}\text { O Em lotes. } \\
\text { O Uma operação após a outra. } \\
\text { O Abordagem "empurra" a produção/ } \\
\text { orientada para a utilização. }\end{array}$ & $\begin{array}{l}\text { O De acordo com a demanda. } \\
\text { O Sobreposição de operações. } \\
\text { Abordagem "puxa" a produção/orien- } \\
\text { tada para o processo. }\end{array}$ \\
\hline \multicolumn{2}{|c|}{ TEMPO REQUERIDO PARA EXECUÇÃO } \\
\hline $\begin{array}{l}\text { OMínimo por operação. } \\
\text { O Máxima produção por minuto. }\end{array}$ & $\begin{array}{l}\text { O Mínimo por ordem. } \\
\text { O Máxima utilização por período. }\end{array}$ \\
\hline \multicolumn{2}{|c|}{ FLUXO DE MATERIAL E INFORMAÇÃO } \\
\hline Consideração de forma isolada. & O Integração. \\
\hline
\end{tabular}

Figura 2. Filosofia de fabricação (CIM e Convencional). Fonte: BULLINGER et alii [5]. 
Uma possivel arquitetura para um Sistema Integrado de Manufatura encontra-se na Figura 3.

Como se pode ver, o sistema é controlado por uma estrutura hierárquica onde um conjunto de computadores interagem enviando instruções de um nível mais alto (um controle gerencial, como definiremos na próxima seção), atéo nível mais baixo (controle físico dos equipamentos) para que as operações sejam realizadas.

A integração necessita de uma estrutura de controle hierarquizada cujos elementos sejam compatíveis e comunicáveis entre si. Assim a interligação dos computadores de controle deve ser feita através de redes locais de interligação (LANs) apropriadas.

Em nível de chãodefábrica aparecem os FMSs ('Flexible Manufacturing Systems'=Sistemas Flexíveis de Manufatura), que são subsistemas vitais do SIM.UmFMSconsiste de um conjunto de máquinas-ferramenta, equipamentos de movimentação de materiais e'facildades" para a armazenagem em processo que estão sob o controle de um sistema de computador [6]. Foram introduzidos por volta de 1970, exigem alto investimento trazem umaumento substancial da produtividade na fabricaçãœm lotes [12].

Os fatores que contribuem para a flexibilidade dos FMSs são:

a) tempos de preparação muito reduzidos;

b) versatilidade dos centros de usinagem dos FMSs para realizar uma grande variedade de operações em uma peça; c) possibilidade de mudar o roteiro de fabricação para contornar o problema de máquinas paradas para reparo;

d) disponibilidade de operações alternativas de forma a balancear a carga das máquinas.

Esse último fator recai num problema de controle da produção. Com as operações alternativas para fins de programação da produção ('scheduling', que mostraremos na próxima seção, é uma atividade de controle da produção), pode-se aliviar as máquinasgargalos levando a um aumento global da produtividade do FMS. Ränky [18] denomina isso de 'scheduling' dinâmico.

Um bom sistema de controle da produção trará como principais benefícios:

a) redução dos estoques de material comprado e de material em processo, de modo que se empregue menor capital de giro e não se ultrapasse o espaço disponivel para estoques nas estações de trabalho, que é muito reduzido nos SIMs;

b) aumento da taxa de produção devido a vários fatores entre os quais a reduçãodo tempo de preparação ('set up') e a melhor utilização da mão-de-obra;

c) maior cumprimento dos prazos de entrega, fornecendo, assim, trunfos de vendas para a empresa;

d) maior eficiência no processo de montagem, uma vez que os itens certos estarão no momento certo na seção de montagem. 


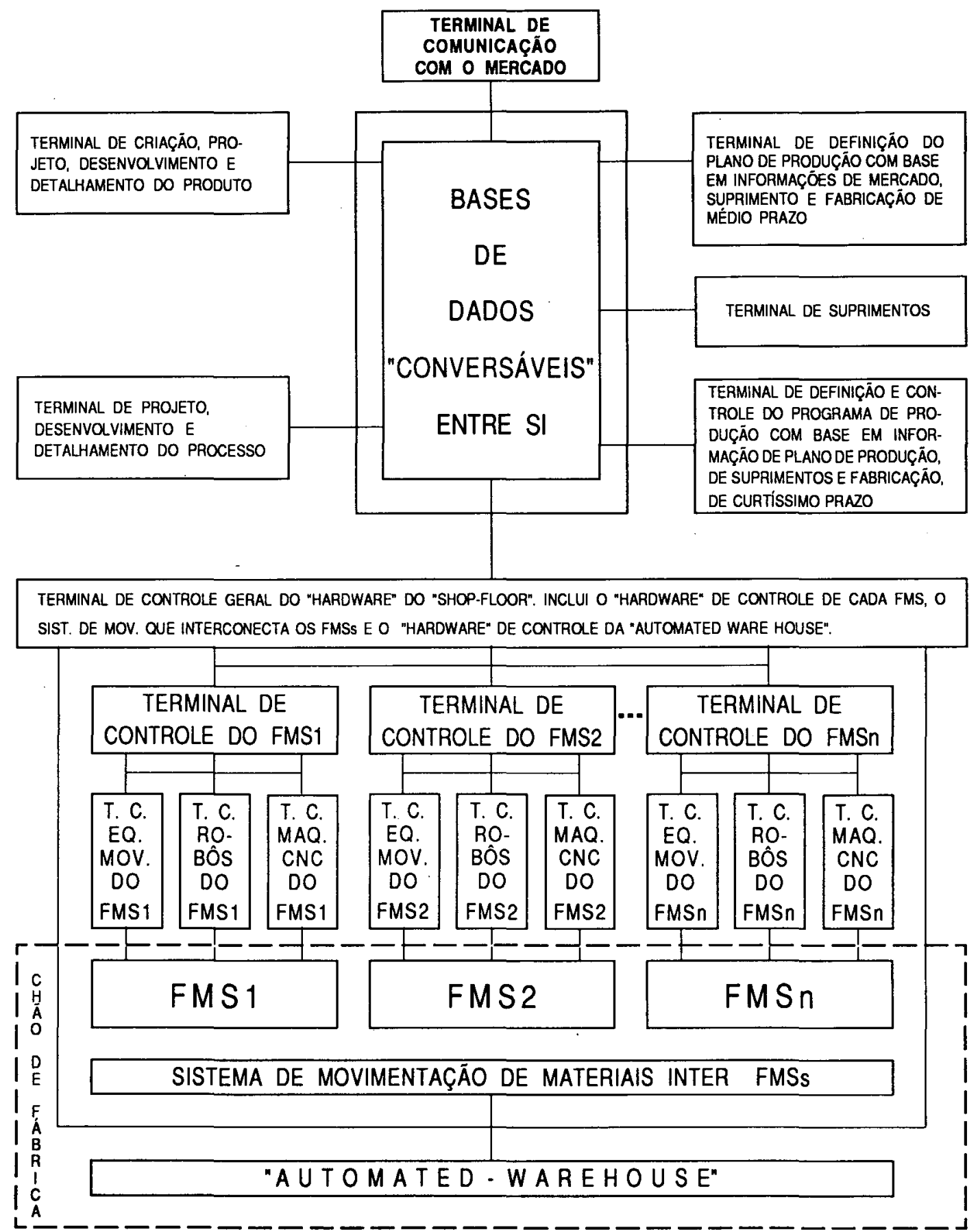

Figura 3. Arquitetura de 'hardware' em nível de integração total (+ nó de rede local de interligação; $t c=$ terminal de controle) 
Do gerenciamento e em particular do controle, muito depende o sucesso de um SIM. Jaikumar [14], comparando sistemas avançados de manufatura americanos e japoneses, afirma que o desempenho dos sistemas japoneses era muito melhor. "Com poucas exceções, os FMSs instalados nos EUA mostram uma alarmante falta de flexibilidade. Em muitos casos, eles têm um desempenho pior que a tecnologia convencional que eles substituíram. A tecnologia em si não é culpada; é o gerenciamento que faz a diferença... As companhias americanas usavam FMSs da forma errada - para alto volume de produção de poucas peças ao invés de grande variedade de peças e baixo custo por unidade" [14]. O mesmo artigo evidencia que Sistemas Flexíveis lembram fábricasem miniatura em operação, e são os labora tórios naturais para estudar a Manufatura Integrada por Computador (CIM) que estão se tornando rapidamente o campo de batalha para obter a supremacia na manufatura em nível mundial.

O sucesso de um SIM depende, em última análise, principalmente da qualificação dos funcionários da engenharia e do gerenciamento da empresa. Numa empresa com funcionários de alto nível, a engenharia cria levando em conta o mercado e o processo, enquanto que a gerência consegue implan- tar sistemas de controle mais eficientes.

Sobre esse último aspecto, o Japão é um bom exemplo. É um país que consegue implantar com sucesso Sistemas Modernos de Gerenciamento, enquanto que, na grande maioria dos países, a implantação é uma fase dificílima. Esse sucesso de implantação, segundo Matsuda [16], advém do que ele chama de inteligência organizacional existente nas fábricas japonesas, e coloca que isso ainda pode ser mais fortalecido pela integração S3: sintese (incentiva coisas diferentes a ficarem juntas), simbiose (incentiva coisas diferentes a viverem juntas para benefício próprio de todas elas) e sinergia (incentiva coisas diferentes a ficarem juntas e obter mais que a simples soma das partes).

A seguir, na Figura 4, esboçamos um modelo conceitual de integração de um Sistema de Manufatura, no qual a função gerencial desempenha um papel preponderante. $O$ controle gerencial capta as necessidades (detonadas a partir do mercado), avalia, coordena as capacidades (da engenharia, do suporte e do chão de fábrica) e exerce um controle sobre o controle físico (das operações e da movimentação dos materiais). Exercer controle sobre o controle pode ser chamado de meta-controle. 


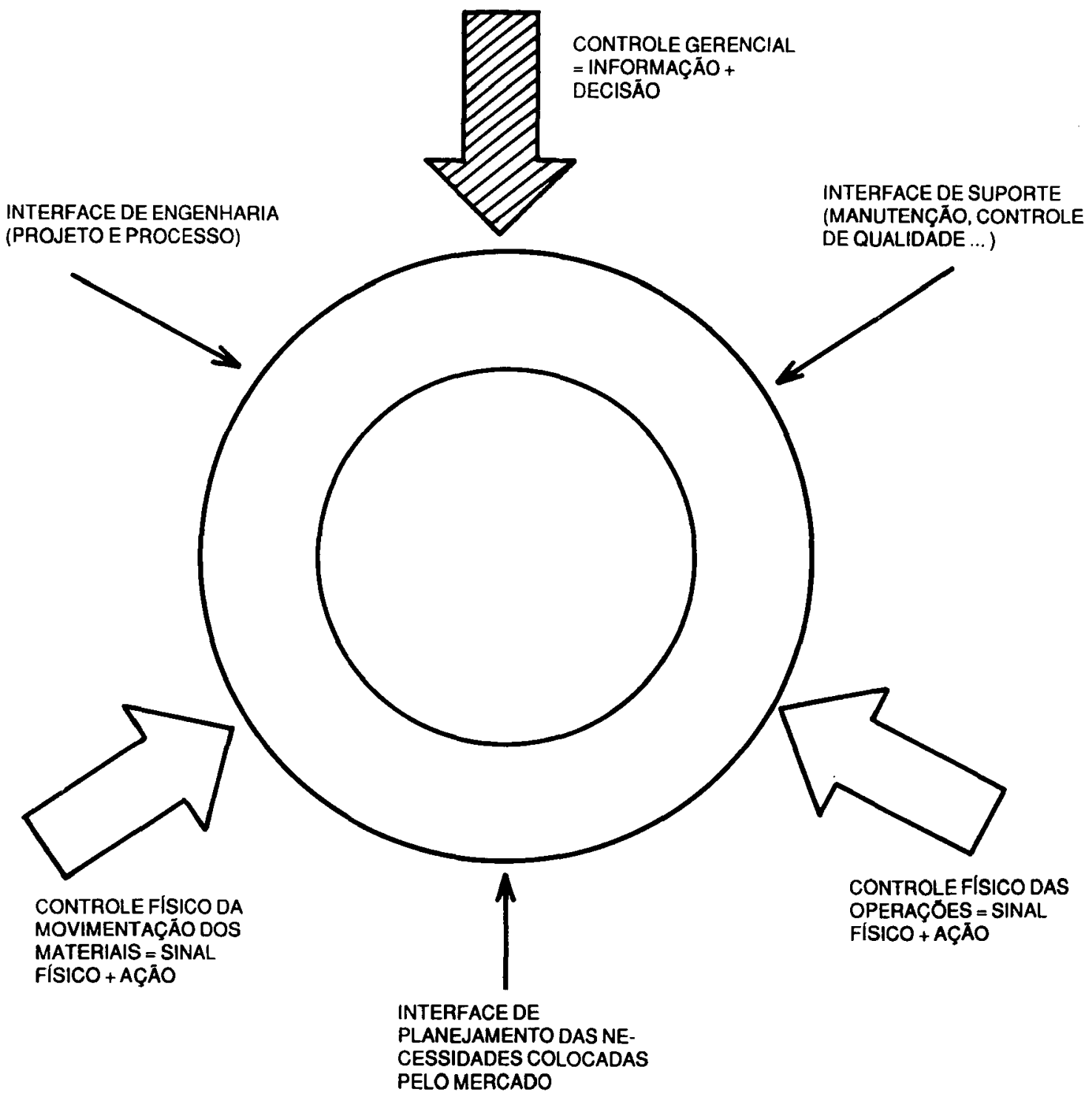

Figura 4. Modelo conceitual de integração de um sistema de manufatura. 


\section{Controle, a Função Vital Para se Obter Integração}

É de conhecimento comum que a atividade de controle é fundamental para a execução de qualquer trabalho. Nos Sistemas Integrados de Manufatura (SIMs) a automação ocupa uma posiçãoda mais alta importância, epor sua vez o controle num ambiente automatizado tem um papel crítico. Para ficar mais claro tomemos a definição encontrada em Goover [11]:

"Automação é a tecnologia relacionada com a aplicação de complexos sistemas mecânicos e eletrônicos, baseados no computador para a operação e o controle da produção. Essa tecnologia inclui:

a) máquinas ferramenta automáticas para processar peças;

b) sistemas automáticos de movimentação de materiais;

c) máquinas para montagem automática;

d) processos contínuos;

e) sistemas de controle com realimentação ('feedback');

f) sistemas de controle do processo por computador;

g) sistemas computadorizados para coleção de dados, planejamento e tomada de decisão para suportar as atividades de manufatura". Nessa definiçã̀o o termo controle aparece explicitamente três vezes, e no último parágrafo ele aparece novamente, porém de forma implícita.

Há algumas décadas Bright [4]demonstrou que a natureza do controle tem íntima relação com os níveis de automação. Um resumo dos 17 niveis de mecanização de Bright encontra-se na Figura 5. 


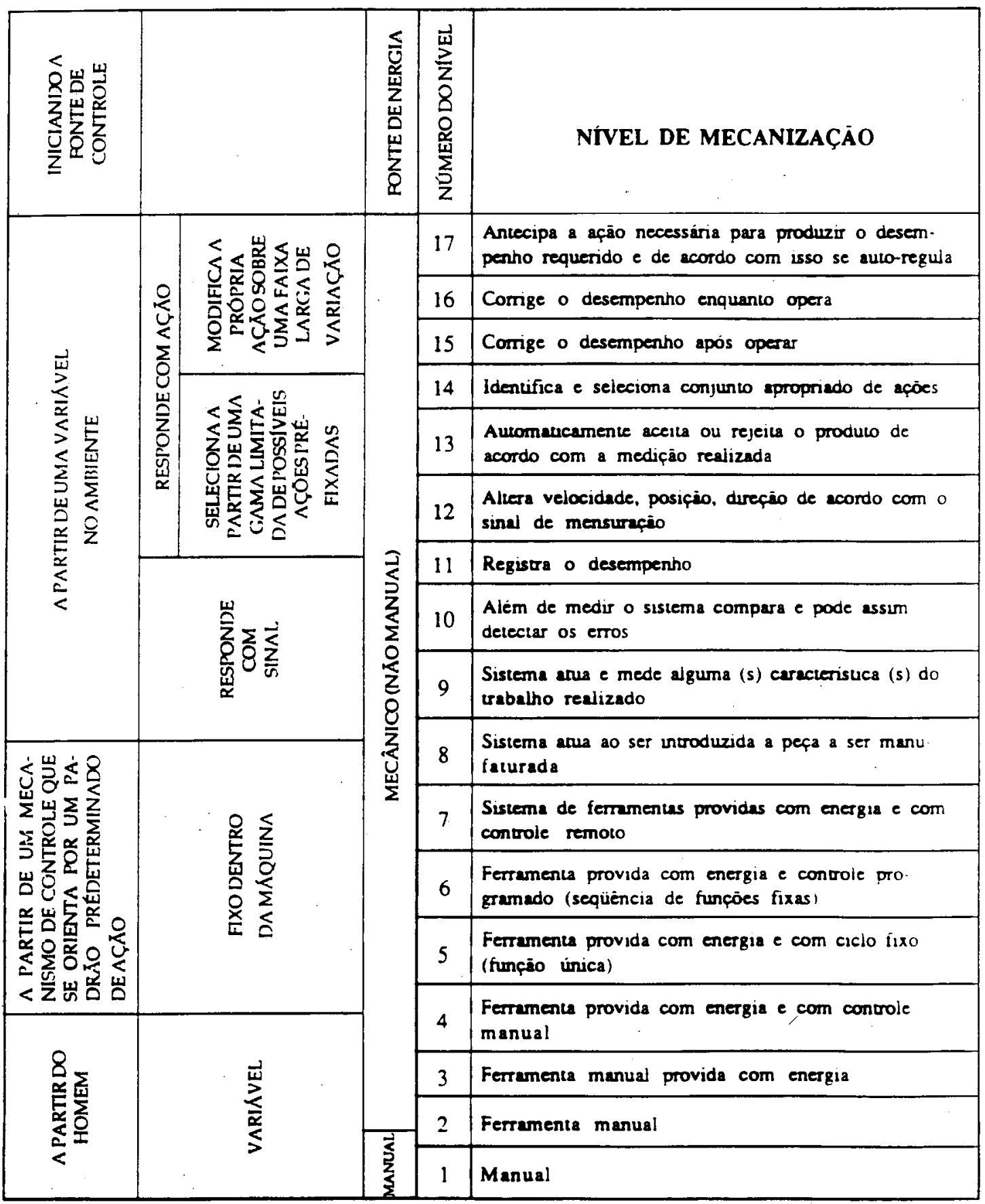

Figura 5. Os 17 niveis de mecanização e suas relações com as fontes de energia e de controle. Fonte: BRIGHT [4]. 
A visão de controle mais estreita é a que o considera apenas como monitoramento. $\mathrm{E}$ talvez a mais abrangenteéa de Leeuw (apud [3]) que entende que qualquer fenômeno de interesse pode ser modelado como uma configuração de controle, que consiste de um controlador, um sistema controlado e um ambiente. Para ele, controle é qualquer forma de influência dirigida do controlador sobre o sistema controlado. Leeuw estabelece que um controlador efetivo deve ter: (a) umobjetivo; (b) um modelo de sistema controlado; (c) informação sobre o ambiente e o estado do sistema controlado; (d) ações adequadas de controle e (e) suficiente capacidade de processamento de informação.
De uma maneira geral, todo controle engloba:

a) a definição de um padrão de resposta do sistema que está sendo controlado. Isso implica um processo de tomada de decisão;

b) a monitoração da resposta para efeito de comparação com o padrão selecionado. No monitoramento, dados sobreo processo são transmitidos para o computador;

c) ação para alterar, se for necessário, osestimulos/entradas/padrõesdeforma a se atingir os resultados desejados (veja Figura 6).

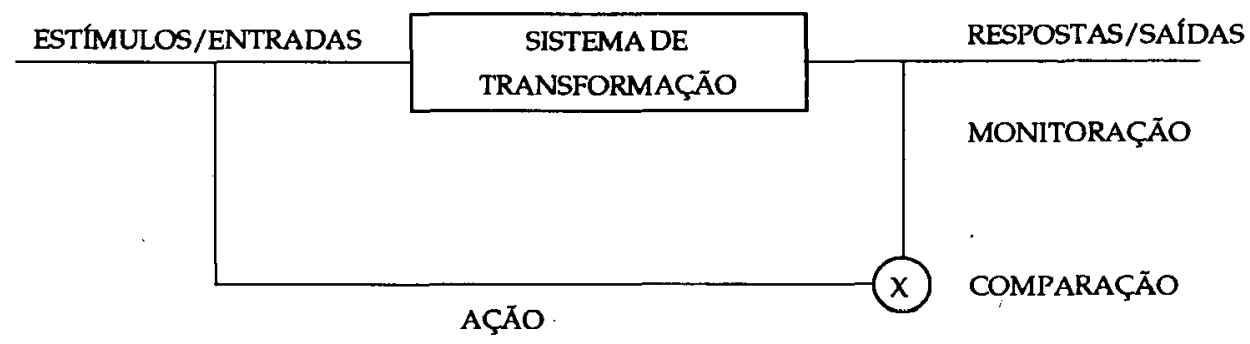

Figura 6. O esquema do processo de controle.

Qualquer sistema "é um conjunto de elementosque atuam juntos na execuçãodoobjetivo global do todo" [7].

Quanto à natureza dos elementos existem os sistemas "físicos" e os sistemas "gerenciais". Nos primeiros os elementos são entidades físicas (por exemplo, um automóvel), enquanto que nos segundos os elementos são procedimentos (por exemplo, os sistemas MRP).

Da mesma forma que num automóvel existem subsistemas, por exemplo o Sistema de Comando de Válvulas para controlar a injeção de combustível nos cilindros, num Sistema de Manufatura também existem subsistemas, por exemplo o Sistema de Informações cha- mado de Sistema de Programação da Produção que irá controlar o fluxo de materiais e a seqüência de operações no chão da fábrica.

Dessa forma podemos depreender dois tipos de controle:

1. controle físico

2. controle gerencial

O controle físico é o controle existente, por exemplo, nogovernador das máquinas a vapor (James Watt, fins do século XVIII), cuja finalidade é manter sob controle o número de rotações por minuto do eixo da máquina. Se a velocidade aumenta, aumenta a força centrífuga sobre duas esferas acopladas ao 
eixo de saída, com isso elas se afastam do eixo, e quanto maior o afastamento mais se fecham as válvulas que controlam a entrada de vapor nos êmbolos, fazendo então a velocidade diminuir em direção ao padrão.

Já o controle da produção, o qual engloba a programação da produção, é um controle tipicamente gerencial. A informação é o "sangue" que flui tanto se osistema émanual (via documentos, tais como ordens de serviço, etc.) ou se é automatizado (via terminais, redes locais de interligação (LAN - 'local area network'), etc.).

Há portanto uma perfeita analogia entre os tipos de sistemas e os tipos de controle. A parte visivel de qualquer sistema de manufatura são subsistemas físicos cuja operação depende de sistemas de controle físicos. Já a integração dos vários subsistemas físicos é feita por sistemas gerenciais que realizam o controle do tipo gerencial. Quanto mais integração for requerida, mais apurado deve ser o controle gerencial.

E mais ainda, existem vários tipos de controles gerenciais de acordo com o problema que ele aborda: controle da produção, controle da produtividade, controle da qualidade, controle de manutenção, controle dos estoques, controle da movimentação dos materiais, etc.

Um parâmetro que é comum a todos esses controles é o tempo. No controle físico o tempo de desencadeamento de uma ação no 'loop' de realimentação (Figura 6) é instantâneo, enquanto que no controle gerencial isso ocorre nogeral em intervalos nítidos de tempo; nesse sentido dizemos que o controle gerencial é geralmente 'off line' enquanto que o controle físico é sempre 'on-line'.

O controle gerencial da produção é o que mais afeta a integração, e a maior atenção damos a ele. Além disso, num SIM o controle da produção e da manutenção passam a ter uma importância muito maior do que têm nos sistemas de fabricação convencionais. As razões disso são que num SIM os equipamentos são caríssimos e assim devem ser cortados ao máximo os tempos improdutivos evitáveis: a diminuição dos tempos de preparação através de uma programação da produção eficiente (que deve levar em conta outros fatores, tais como prazos de entrega e inventários em processo) e a diminuição da ociosidade das máquinas através de uma programação da manutenção que preveja reparos preventivos exatamente quando o equipamento não estiver sendo usado pela programação da produção.

O acompanhamento da produção deve ser capaz de coletar os dados certos na hora certa sobre o que está ocorrendo no chão da fábrica de forma que as reprogramações da produção e da manutenção sejam feitas com dados atualizados.

O controle da produção compreende:

a) definir (estabelecer padrão) o que deve ser feito em cada estação de trabalho numa base de tempo de curto prazo (programação da produção = 'scheduling');

b) monitorar o que está acontecendo no chão da fábrica ('shop-floor control');

c) comparar o que está acontecendo com o programado e, em casos de desvios, realimentar o processo através de uma reprogramação.

A fase (a) ou seja a programação da produção, é basicamente um processo de tomada de decisão. É aí que metodologias como Pesquisa Operacional (PO) e mais recentemente Inteligência Artificial (IA) têm importância no controle da produção, principalmente na Manufatura Integrada. A relevância da Pesquisa 
Operacional no controle dos SIMs será objeto de exploração num outro artigo.

\section{Considerações Finais}

Os fatores estratégicos para uma empresa de manufatura se manter no mercado futuro são: adaptabilidade (capacidade da engenharia de projeto criar e desenvolver produtos na velocidade que o mercado consumidor demande), flexibilidade (capacidade do processo de fabricação se adaptar a tempo para produzir os novos produtos introduzidos), qualidade e produtividade [9].

Não se pode ter uma dosagem apropriada de adaptabilidade, flexibilidade, produtividade e qualidade sem haver integração. Empresas não integradas tendem a ser fortes em aspectos não tão essenciais e fracas em aspectos cruciais para as suas características de relacionamentocomomercadoconsumidor. Por exemplo, ser apenas altamente produtiva quando sua situação exige alta adaptabilidade.

Como vimos essa integração depende basicamente dos controles gerenciais.

Uma tendência marcante nos SIMs que estão sendo desenvolvidos nos países mais adiantados é que está havendo uma centralização do controle, algo que foi previsto há três décadas. Em 1958, Leavitt e Whisler [15] previram que na década de 80 , com a combinação da MS ('Management Science' = PO no âmbito do gerenciamento) e da Tecnologia da Informação, haveria uma descentralização em nivel de tomada de decisão, uma centralização em nível de controle, uma redução grande do número de gerentes médios e maior controle da alta gerência sobre a empresa. Essa descentralização em nivel de tomada de decisão deve se tornar maior à medida que os DSSs
('Decision Support Systems' = Sistemas de Apoioà Decisão) vão sendo desenvolvidos e implantados. Além disso os computadores estão assumindo muitas funções de controle que cabiam aos gerentes médios. Os gerentes de linha, que estão bem como a alta gerência, estão passando a ter uma responsabilidade maior.

Acreditamos que essa maior centralização docontroleé fruto do desenvolvimento da tecnologia da informação, enquanto que a descentralização da tomada de decisões está se tornando fruto do desenvolvimento do DSS que é um tipo de SI (Sistema de Informaçãoque faz uso das técnicas de MIS (Sistemas de Informações Gerenciais), das de PO (Pesquisa Operacional) e das de IA (Inteligência Artificial), e não descarta, na solução de problemas não-estruturados ou até mesmo semiestruturados, a participação da gerência.

Applegate et alii [1] acreditam que o efeito dessas tecnologias será o aparecimento deuma nova forma organizacional, a organização 'cluster' (organização de grupos), onde os grupos de pessoas, mesmo fisicamente distanciadas, trabalham juntas para resolver os problemas da companhia ou para definir um processo. Assim os sistemas de informação e comunicação permitirão às pessoas com habilidades que se complementam, trabalharem juntas.

Para finalizar, enfatizandoa importância do controle para a empresa industrial, citamos Beer [2] que mostra a correspondência existente entre o sistema de controle de uma empresa e o sistema nervoso central do corpo humano: “... existem óbvias semelhanças entre os controles usados numa empresa e aqueles usados no corpo humano. Por exemplo: ambos são hierárquicos, ambos são redundantes e ambos incorporam subsistemas de maior ou menor autonomia". 


\section{Referências Bibliográficas}

[1] APPLEGATE, L., CASH JR, J., MILLS, Q. "Information Technology and Tomorrow's Manager", Harvard Business Review, pp. 128-136, nov-dec. 1988.

[2] BEER, S., Decision and Control, Wiley, 1966.

[3] BOER, H., DURING, W.E. "Management of process innovation the case of FMS a system approach", Int. J. Production Res., vol. 25, n.o 11, pp. 1671-1682, 1987.

[4] BRIGHT, J.R. Automation and Management, Harvard University, 1958.

[5] BULLINGER, H.J., WARNECKE, H.S., LENTIS, H.P. "Toward the factory of the Future", Int. J. Production Res., vol. 24, n.o 4, pp.697-741, 1986.

[6] BUZACOTT, J.A., SHANTHIKUMAR, J.G. "Models for Understanding Flexible Manufacturing Systems, AIIE Transation, Dec. 1980.

[7] CHURCHMAN, C.W. Introdução à teoria dos sistemas, Ed. Vozes, 1972.

[8] FERDOWS, K., LINDBERG, P. "FMS as Indicator of the Strategic Role of Manufacturing", Int. J. of Production Res., vol. 25, n.o 11, pp. 1563-1571, 1987.

[9] GERELLE, E.G.R., STARK, J. Integrated Manufacturing Strategy, Planning and Implementation, McGraw-Hill, 1988.
[10]GOLDRATT, E., COX, J. A Meta, IMAM (Instituto de Movimentação e Armazenagem de Materiais).

[11] GROOVER, M.P. Automation, Production Systems and Computer-Aided Manufacturing, Prentice-Hall, 1980.

[12] GROOVER, M.P., ZIMMERS JR., E.W. CAD/CAM: Computer-Aided Design and Manufacturing, Prentice Hall, 1984.

[13]HITOMI, K. Manufacturing Systems Engineering, Taylor \& Francis, London, 1979.

[14]JAIKUMAR, R. "Postindustrial Manufacturing". Harvard Business Review, pp.69-76, nov.-dec. 1986.

[15] LEAVITT, H.J., WHISLER, T.L. "Management in the 1980 's", Harvard Business Review, nov.-dec. 1958.

[16] MATSUDA, T. “OR/MS, its Interaction with and Benefit from Japanese Organizational Intelligence", Omega, vol. 16. n.o 3, pp. 233-141, 1988.

[17] MERCHANT, M.E. "Delphi-type Forecast of the Future of Production Engineering", Annals of CIRP, 20(3), pp. 213-225, 1971.

[18] RÄNKY, P. The Designand Operation of FMS - Flexible Manufacturing Systems, IFS (Publications)/North Holland Publ., 1983.

[19] SINGHAL, K., FINE, C.H., MEREDITH, J.R., SURY, R. "Research and Models for Automated Manufacturing", Interfaces, vol. 17, n.o 6, pp. 5-14, nov.dec. 1987. 